\title{
SYNTHESIS OF NITROGEN-RICH GaNAS SEMICONDUCTOR ALLOYS AND ARSENIC-DOPED GaN BY METALORGANIC CHEMICAL VAPOR DEPOSITION
}

\author{
M. Gherasimova, B. Gaffey, P. Mitev, and L. J. Guido \\ Department of Electrical Engineering, Yale University, Box 208284, New Haven, CT 06520 \\ K. L. Chang and K. C. Hsieh \\ Department of Electrical and Computer Engineering, University of Illinois, 1406 W. Green St., Urbana, IL 61801
}

S. Mitha

Charles Evans \& Associates, 301 Chesapeake Dr., Redwood City, CA 94063

J. Spear

Philips Electronic Instruments, 125 West Gemini Dr., Tempe, AZ 85283

Cite this article as: MRS Internet J. Nitride Semicond. Res. 4S1, G3.44(1999)

\begin{abstract}
Arsenic-doped GaN films and GaNAs films have been synthesized by MOCVD. Samples were grown on sapphire, GaN-coated sapphire, and GaAs substrates. Composition, structure, and phase distribution were characterized by EPMA, SIMS, XRD, and TEM. The arsenic content increases demonstrably as the growth temperature descreases from 1030 to $700{ }^{\circ} \mathrm{C}$. In the high temperature limit, high quality arsenic-doped GaN forms on GaN-coated sapphire. In the low temperature regime, nitrogen-rich GaNAs forms under some growth conditions, with a maximum arsenic mole fraction of 3\%, and phase segregation in the form of GaAs precipitates occurs with an increase in arsine pressure. Preferential formation of the nitrogen-rich phase on GaN-coated sapphire suggests the presence of substrate-induced "composition pulling".
\end{abstract}

\section{INTRODUCTION}

Group III nitride semiconductor alloys with mixing on the anion sublattice are of considerable interest because of their potential application to a wide range of optoelectronic devices. The large difference in bond lengths between group III nitrides and arsenides results in an enormous miscibility gap in the pseudo-binary phase diagram, making it difficult to synthesize alloys in the intermediate composition range. While arsenic-rich alloys have been studied by a number of research groups, there are only a few reports on the synthesis of nitrogen-rich compounds. ${ }^{1-3}$ In the present work, nitrogen-rich GaNAs alloys and arsenic-doped GaN have been synthesized by metalorganic chemical vapor deposition (MOCVD). Emphasis was placed on determining the influence of growth parameters, and the choice of substrate material, on the composition, phase distribution, and structural properties of these materials.

\section{EXPERIMENTAL PROCEDURE}

All of the samples described herein were grown in a conventional, horizontal-flow MOCVD reactor held at a pressure of 100 torr. Palladium-purified hydrogen was used as the carrier gas with a total flow rate of $8 \mathrm{slm}$. Trimethylgallium, ammonia, and arsine precursors were employed during growth, with the ammonia and trimethylgallium flows kept constant at $4 \mathrm{slm}$ and $50 \mu \mathrm{mol} / \mathrm{min}$, for all samples, while the arsine flow was varied between 4 and $400 \mathrm{sccm}$. Growths were performed on bare sapphire and GaAs substrates, as well as on $\mathrm{GaN}$ pseudo-substrates, ${ }^{4}$ for the purpose of studying the relationship between gas phase chemistry and arsenic incorporation in the solid. In addition, a two-step growth method was employed in some cases to optimize electronic properties. ${ }^{5}$ Film composition was determined using quantitative electron probe microanalysis (EPMA), by measuring the nitrogen $\mathrm{K} \alpha$ line and gallium and arsenic $\mathrm{L} \alpha$ line intensities, with the electron beam energy ranging from 6 to $13 \mathrm{keV}$. Substrate and surface contamination effects were eliminated as sources of error by performing variable-energy measurements. The arsenic mole fraction (x) and III-V stoichiometry ratio (s) were derived as follows

$$
\mathrm{x}=\frac{[\text { As atomic } \%]}{[\text { As atomic } \%]+[\mathrm{N} \text { atomic } \%]} \quad ; \quad \mathrm{s}=\frac{[\mathrm{Ga} \text { atomic } \%]}{[\text { As atomic } \%]+[\mathrm{N} \text { atomic } \%]}
$$


Secondary ion mass spectroscopy (SIMS) analysis was also performed for quantification purposes, with the $\mathrm{CsM}^{+}$ molecular ion technique used for GaNAs alloys, ${ }^{6}$ and an arsenic implant standard employed to calibrate arsenic doping. Transmission electron microscopy (TEM) and $\mathrm{x}$-ray diffraction (XRD) measurements were performed to evaluate the structural properties of these materials.

\section{RESULTS}

\section{A. Effects of Growth Temperature on Arsenic Incorporation}

The general trend observed in all experiments is that an increase in growth temperature leads to a decrease in arsenic incorporation. Table I summarizes our observations for samples grown with an arsine flow of $4 \mathrm{sccm}$. These films were deposited on $\mathrm{GaN}$ pseudo-substrates, except for the $1030^{\circ} \mathrm{C}$ sample which was grown using the two-step method. At 950 and $1030{ }^{\circ} \mathrm{C}$ the arsenic content is below the EPMA detection level so SIMS analysis was used for quantification.

Table I. Arsenic incorporation dependence on growth temperature.

\begin{tabular}{cccc}
\hline \hline Temperature $\left({ }^{\circ} \mathrm{C}\right)$ & Composition $(\%)$ & Measurement & Stoichiometry (s) \\
\hline 700 & $3.2 \pm 0.3$ & EPMA & $0.98 \pm 0.02$ \\
800 & $0.19 \pm 0.02$ & EPMA & $0.96 \pm 0.02$ \\
900 & $0.10 \pm 0.04$ & EPMA & $0.98 \pm 0.02$ \\
950 & 0.02 & SIMS & $0.99 \pm 0.01$ \\
1030 & $2 \times 10^{16} \mathrm{~cm}^{-3}$ & SIMS & $0.97 \pm 0.02$ \\
\hline \hline
\end{tabular}

\section{B. Effects of Substrate Material on Phase Separation}

The phase distribution of GaNAs films grown at $700{ }^{\circ} \mathrm{C}$ exhibits a strong dependence on the choice of substrate. Phase segregation was observed in some samples, Table II, with multi-faceted GaAs particles (average size $\approx 10-30$ $\mu \mathrm{m})$ precipitating on top of specular GaNAs films. For cases were more than one phase is present, EPMA measurements were conducted on all visually distinct features. The influence of substrate in the present work is consistent with the well-documented composition pulling phenomenon ${ }^{7}$ (e. g., the trend for samples grown with 4 sccm of arsine).

\section{High Temperature Growth of Arsenic-Doped Gallium Nitride}

Previously we demonstrated that arsenic doping significantly improves the electronic properties of GaN epitaxial layers. ${ }^{8}$ The present work confirms our original findings, and provides additional data on the chemical and structural properties of these materials. At a growth temperature of $1030{ }^{\circ} \mathrm{C}$, the arsenic doping level increases from $2 \times 10^{16}$ to $2 \times 10^{17} \mathrm{~cm}^{-3}$ in

Table II. Phase distribution dependence on substrate and arsine flow.

\begin{tabular}{cccc}
\hline \hline $\begin{array}{c}\mathrm{AsH}_{3} \text { flow } \rightarrow \\
\text { Substrate } \downarrow\end{array}$ & $4 \mathrm{sccm}$ & $40 \mathrm{sccm}$ & $400 \mathrm{sccm}$ \\
$\mathrm{GaN}$ & GaNAs & GaNAs $+\mathrm{GaAs}$ & $\mathrm{GaAs}$ \\
$\mathrm{Al}_{2} \mathrm{O}_{3}$ & $\mathrm{GaNAs}+\mathrm{GaAs}$ & $\mathrm{GaAs}$ & $\mathrm{GaAs}$ \\
$\mathrm{GaAs}$ & $\mathrm{GaAs}$ & $\mathrm{GaAs}$ & $\mathrm{NA}$ \\
\hline \hline
\end{tabular}


response to a change in arsine pressure from 0.03 to 3.23 torr. In addition, no appreciable difference in arsenic concentration is found for samples deposited on GaN pseudo-substrates as compared to those prepared using the two-step growth method. Figure 1 shows that the residual impurity content does not change significantly as arsine is added to the growth chemistry. In particular, the oxygen concentration does not increase with arsenic incorporation - which supports the claim that the arsine cylinder was the source of oxygen contamination in our original experiment.

High-resolution x-ray diffraction measurements were performed to determine the influence of arsine on macroscopic film structure. In particular, diffraction space maps were obtained for the symmetric (004) and asymmetric (101) reflections - the omega scan component of these maps provides a measure of epitaxial layer tilt and column twist. Table III gives the full-width at half-maximum values derived from these measurements for both a conventional high-quality GaN layer and an arsenic-doped film with $[A s]=2 \times 10^{17} \mathrm{~cm}^{-3}$. Lateral coherence length measurements were also made using the (104) reflection to estimate the average grain size.

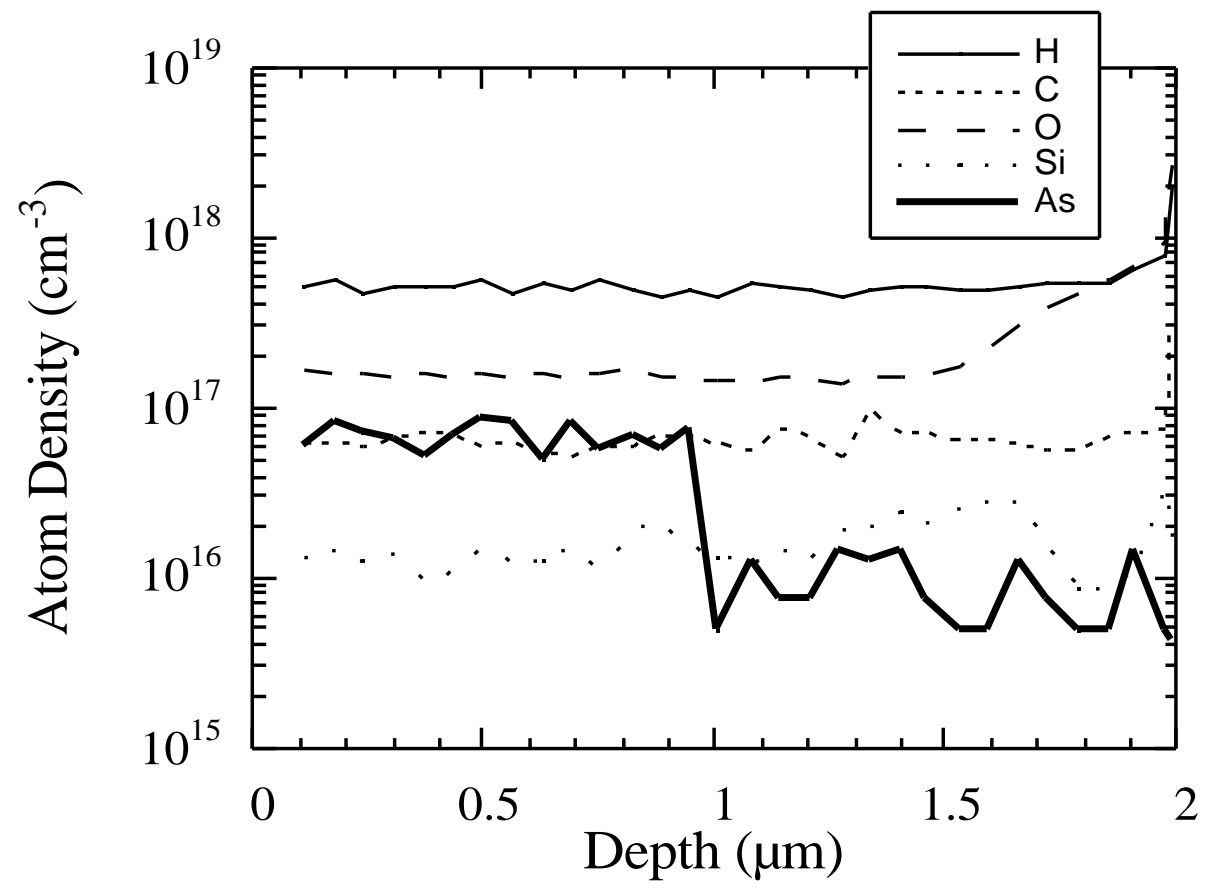

Figure 1. SIMS depth profile for $1 \mu \mathrm{m} \mathrm{GaN}$ :As film grown on GaN pseudo-substrate. Arsine pressure during the growth of the doped layer $=0.33$ torr.

Table III. Summary of XRD measurements on high-temperature films.

\begin{tabular}{ccc}
\hline \hline Physical Property & Undoped GaN & Arsenic-doped GaN \\
\hline Layer Tilt (degrees) & 0.101 & 0.101 \\
Column Twist (degrees) & 0.129 & 0.273 \\
Coherence Length $(\AA)$ & $7750 \pm 150$ & $8200 \pm 200$ \\
\hline \hline
\end{tabular}

\section{Low Temperature Growth of Nitrogen-Rich GaNAs Alloys}

A series of samples has been grown on $\mathrm{GaN}$ pseudo-substrate at $700^{\circ} \mathrm{C}$, with the arsine flow varying from 0 to 400 $\mathrm{sccm}$. The GaN control sample has a rough surface, while the introduction of $4 \mathrm{sccm}$ arsine yields a specular film containing approximately $3 \%$ of arsenic. A further increase in arsine flow to $40 \mathrm{sccm}$ leads to the formation of multi-faceted GaAs precipitates; however, EPMA analysis shows that the composition of the GaNAs region saturates at $3 \%$. 


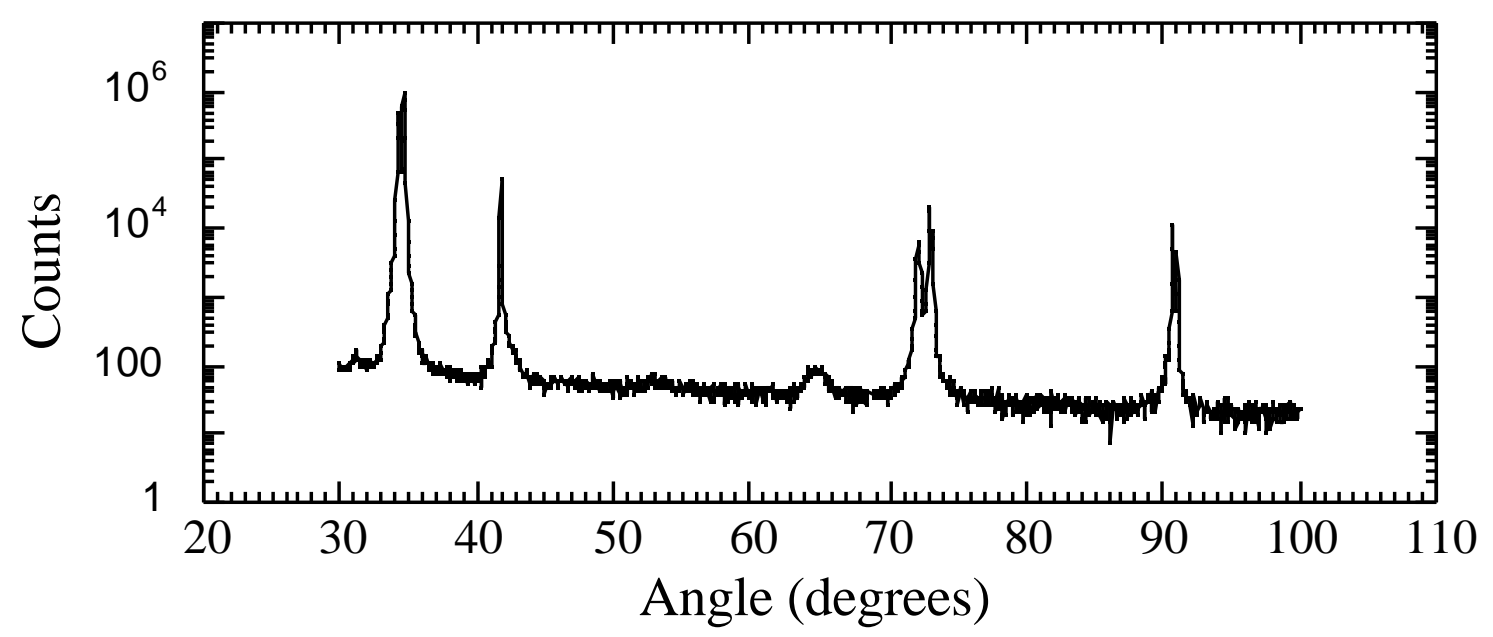

Figure 2. X-ray powder diffraction scan for GaNAs sample grown at $700^{\circ} \mathrm{C}$.

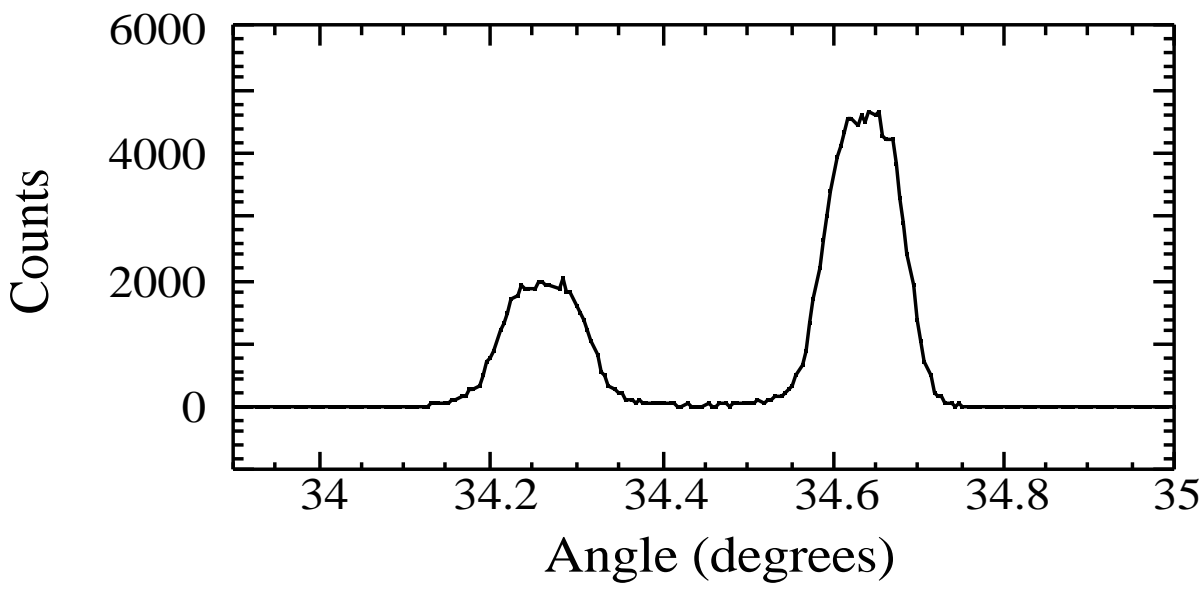

Figure 3. High resolution x-ray rocking curve showing (002) GaN and GaNAs peaks.

Finally, at $400 \mathrm{sccm}$ of arsine, a rough but continuous GaAs film is synthesized with the nitrogen content reduced to below the SIMS detection limit. Figure 2 shows an x-ray powder diffraction scan for a sample prepared using 4 sccm of arsine. The strong peak at $34.6^{\circ}$ is caused by the diffraction from the (002) planes of the GaN pseudosubstrate.

A high resolution x-ray rocking curve about the (002) GaN peak is shown in Figure 3. The second peak at smaller diffraction angle $\left(34.2^{\circ}\right)$ is attributed to GaNAs with approximately $4 \%$ arsenic mole fraction, assuming that the GaNAs layer is pseudomorphic with GaN. If instead the GaNAs is presumed to be fully relaxed the diffraction peak separation suggests an arsenic content of almost $8 \%$.

TEM measurements were also performed to examine the microstructure of these GaNAs films. The crosssectional image shown in Figure 4 is representative of films grown at $700^{\circ} \mathrm{C}$ with 4 and $40 \mathrm{sccm}$ of arsine. There are two particularly important features to notice. First, the landscape is separated into vertical columns that are roughly $250 \AA$ wide. These same features are observed in a control sample (without arsenic) that consists of a $700^{\circ} \mathrm{C} \mathrm{GaN}$ layer deposited on a GaN pseudo-substrate. The origin of these columns is most likely the three-dimensional mode of growth by which GaN islands nucleate on the substrate surface. As the growth proceeds, these islands grow in size and eventually coalesce to form a contiguous film. Such columns with a large vertical-to-horizontal aspect ratio are expected to form if the vertical growth rate is much greater than its lateral counterpart. The initial areal density of nuclei decreases exponentially with an increase in growth temperature, and, as a consequence, the final width of the column just before coalescence is much larger in samples grown at higher temperature. This viewpoint is consistent with the absence of column boundaries in the GaN pseudo-substrate region of Figure 4, since their average width is expected to be larger than the length scale of the image. Second, there are many distinct changes in contrast along the length of each column, some of which may be caused by phase separation into nitrogen- and 
arsenic-rich regions, and others which are indicative of the presence of stacking faults. By comparison, the $700^{\circ} \mathrm{C}$ $\mathrm{GaN}$ control sample exhibits considerably fewer contrast fringes along the length of each column.

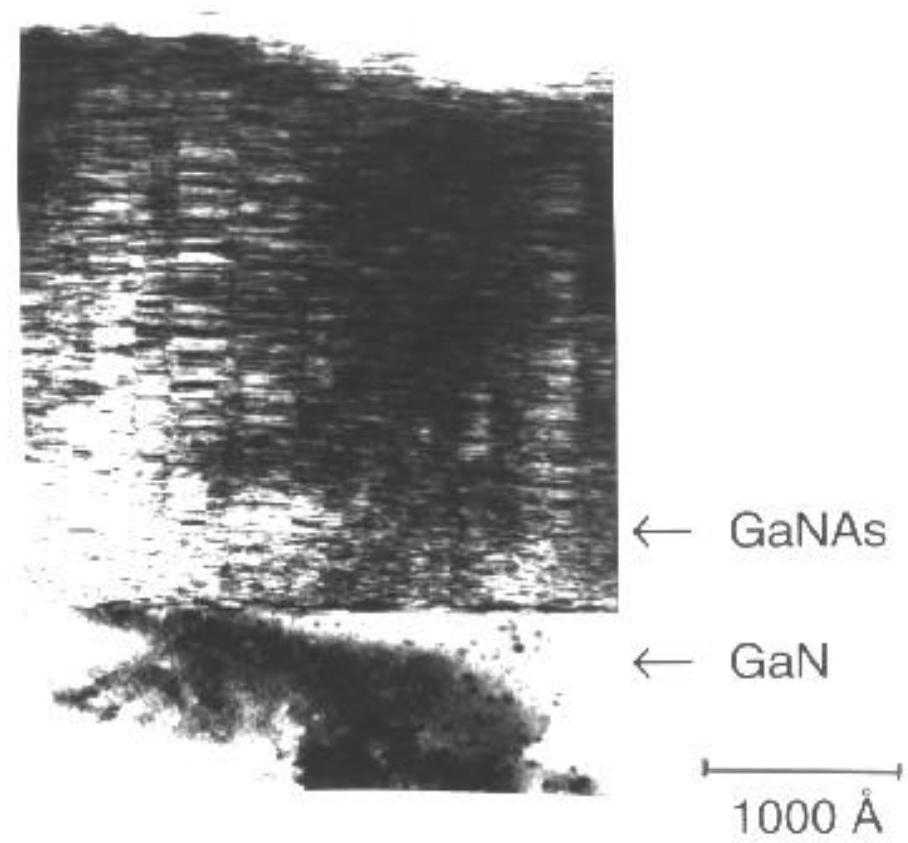

Figure 4. Cross-sectional TEM image : GaNAs film grown at $700^{\circ} \mathrm{C}$ on $\mathrm{GaN}$ pseudo-substrate.

\section{SUMMARY \& CONCLUSIONS}

Arsenic-doped GaN and nitrogen-rich GaNAs films have been synthesized using a conventional MOCVD reactor with trimethylgallium, ammonia, and arsine precursors. Direct deposition of $\mathrm{GaN}$ on sapphire at $1030^{\circ} \mathrm{C}$ in the presence of arsine causes a significant reduction in growth rate. The growth rate of undoped GaN is $2.4 \mu \mathrm{m} / \mathrm{h}$, which is similar to that if $\mathrm{GaN}$ deposited via the two-step method, but it decreases to $1.7 \mu \mathrm{m} / \mathrm{h}$ at an arsine pressure of 0.33 torr. Complete growth inhibition occurs at an arsine partial pressure of 3.23 torr. This observation suggests that arsenic may have a strong surfactant effect. The nitrogen content is below the SIMS detection limit for films deposited directly on GaAs substrates, which suggests the presence of strong composition pulling. All arseniccontaining films deposited on GaN-coated sapphire at $4 \mathrm{sccm}$ of arsine are continuous and specular - with the notable exception of the sample grown at $900^{\circ} \mathrm{C}$, which is very rough relative to those grown at both higher and lower temperatures. This may be indicative of a change in the mode of growth or crystal structure (zincblende vs. wurtzite). At the high-temperature limit $\left(1030^{\circ} \mathrm{C}\right)$, arsenic-doped $\mathrm{GaN}$ is formed with significantly better structural and electronic properties than otherwise identical samples without arsenic. At the low-temperature limit $\left(700^{\circ} \mathrm{C}\right)$, non-homogeneous films are formed with GaNAs alloy regions containing stacking faults surrounded by GaAs (or perhaps arsenic-rich alloy inclusions).

\section{ACKNOWLEDGMENTS}

Funding for ongoing research at the Yale University Optoelectronic Materials and Devices Laboratory has been provided by the National Science Foundation (ECS-92537600). M. Gherasimova and B. Gaffey are grateful for individual financial support provided by an NEC Graduate Student Fellowship and the Fannie and John Hertz Foundation, respectively. Authors gratefully acknowledge the assistance with the EPMA measurements provided by Dr. J. Eckert at the Yale University Geology Department.

\section{REFERENCES}


${ }^{1}$ K. Iwata, H. Asahi, K. Asami, R. Kuroiwa, and S. Gonda, Jpn. J. Appl. Phys. 37, 1436 (1998).

${ }^{2}$ R. Kuroiwa, H. Asahi, K. Asami, S.-J. Kim, K. Iwata, and S. Gonda, Appl. Phys. Lett. 73, 2630 (1998).

${ }^{3}$ Y. Zhao, 1998 Electronic Materials Conference, Charlottesville, June, 1998.

${ }^{4}$ Sapphire substrate coated with a high-quality, $1 \mu \mathrm{m}$ thick GaN epitaxial layer.

$5300 \AA$ of $\mathrm{GaN}$ deposited on sapphire at $550{ }^{\circ} \mathrm{C}$, followed by an arsenic-bearing test layer.

${ }^{6}$ The basic method is described in J. Appl. Phys. 64, 3760 (1988). In the present case, the arsenic mole fraction was obtained by dividing the $(\mathrm{CsAs})^{+}$ion counts by the sum of the $(\mathrm{CsAs})^{+}$and $(\mathrm{CsN})^{+}$signals.

${ }^{7}$ K. Hiramatsu, Y. Kawaguchi, M. Shimizu, N. Sawaki, T. Zheleva, R. F. Davis, H. Tsuda, W. Taki, N. Kuwano, and K. Oki, MRS Internet J. Nitride Semicond. Res., Vol. 2, Article 6.

${ }^{8}$ L. J. Guido, P. Mitev, M. Gherasimova, and B. Gaffey, Appl. Phys. Lett. 72, 2005 (1998). 